\title{
Pleistocene hares from the East Siberian Arctic (Lagomorpha: Leporidae)
}

\section{Alexander O. Averianov, Tatyana V. Kuznetsova \& Pavel A. Nikol'skii}

\begin{abstract}
Isolated fossil hare bones from seven Pleistocene localities in the east Siberian Arctic are attributed to Lepus tanaiticus vereschagini Averianov, 1995 (Mamontovaya Khayata) and to L. tanaiticus subsp. indet. (other localities).
\end{abstract}

KEY-WORDS: Lepus, Pleistocene, Siberia, Arctic.

AlexanderO.Averianov [lepus@zin.ru],Zoological Institute, Russian Academy of Sciences, Universitetskaya nab. 1, Saint Petersburg 199034, Russia; Tatyana V. Kuznetsova [tatkuz@orc.ru], M.V. Lomonosov Moscow State University, Vorob'evy Gory, Moscow 119899, Russia; Pavel A. Nikol'skii [nikol@geo-tv-sign.ru], Geological Institute, Russian Academy of Sciences, Pyzhevskii per. 7, Moscow 119017, Russia.

\section{Плейстоценовые зайцы Восточно-Сибирской Арктики (Lagomorpha: Leporidae)}

\section{А.О. Аверьянов, Т.В. Кузнецова, П.А. Никольский}

РЕЗЮМЕ. Изолированные ископаемые остатки зайцев из семи плейстоценовых местонахождений Восточно-Сибирской Арктики отнесены кLepus tanaiticus vereschagini Averianov, 1995 (Мамонтовая Хаята) и к L. tanaiticus subsp. indet. (другие местонахождения).

КЛЮЧЕВЫЕ СЛОВА: Lepus, плейстоцен, Сибирь, Арктика.

\section{Introduction}

White hare is one of the typical elements of the modern Arctic theriofauna and was very abundant in the Pleistocene periglacial Arctic-like steppes. In Siberia hare remains are quite common in both Paleolithic sites and natural permafrost localities (see review in Averianov, 1995). However, little taxonomic work was done on these materials. Earlier authors usually referred this hare to the modern species Lepus timidus Linnaeus, 1758. Later some authors referred it to the extinct Lepus tanaiticus Gureev, 1964, described previously from the Late Pleistocene of European Russia (Gureev, 1964). Averianov (1995) confirmed the latter referral and established a new subspecies, L. tanaiticus vereschagini Averianov, 1995 for the Late Pleistocene hares from continental Yakutia. He studied also materials from the Laptev Sea coast and Bol'shoi Lyakhovskii Island, but taxonomic attribution of these samples was not clarified, mostly because of lack of diagnostic remains, notably dentaries and anterior premolars (P2 and p3). Here a new material from the Laptev Sea area is described and taxonomic attribution of these hares is discussed.

\section{Material and localities}

Majority of the materials was collected during the field work (1998-2000) of the Russian-German expedition in the frame of the "Laptev Sea System 2000" project (Kuz'minaet al., 1999; Kuznetsova \& Kuz'mina, 2000, 2001). Additional specimens were provided by Pavel A. Nikol'skii (Geological Institute RAS, Moscow). All of these materials are temporarily housed in the Paleontologicheskii Institute RAS in Moscow (abbreviated PIN) and published with field numbers. For comparison the Pleistocene hare bones from Siberia and European Russia, published previously by Averianov \& Kuz'mina (1993) and Averianov (1995, 1999), as well as materials on the modern Arctic hares from the collec-

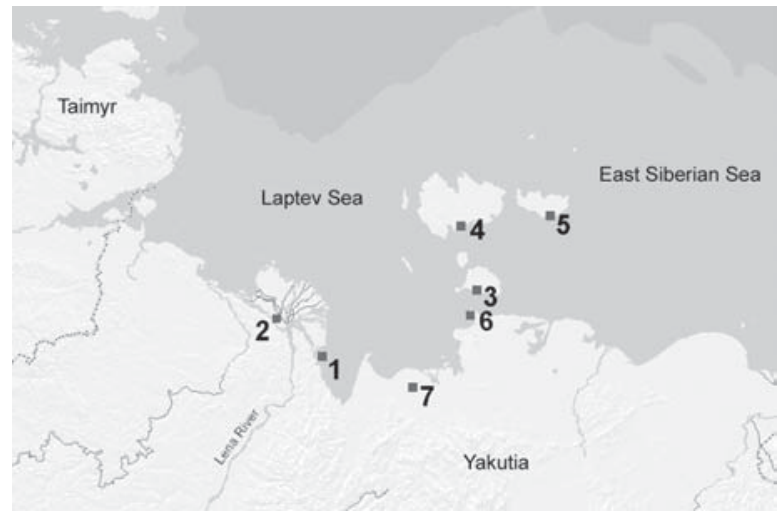

Figure 1. Map of the East Siberian Arctic with position of the Pleistocene hare localities studied in this paper: 1 - Mamontovaya Khayata; 2 - Buor-Khaya; 3 - Bol'shoi Lyakhovskii Island; 4 - Balyktakh; 5 - Tordokh; 6 - Svyatoi Nos; 7 Yana River Paleolithic Site. 
tion of Zoological Institute RAS, were used. Altogether 116 hare bones from seven localities (Fig. 1) were studied:

Locality 1. Mamontovaya Khayata.

Position. Bykovsky Peninsula, Laptev Sea coast, northern Yakutia.

Age. Late Pleistocene.

Material. Four p3, one P2, one skull fragment, five dentary fragments, 35 postcranial bones.

Collector. T.V. Kuznetsova, 1998, 2000.

Locality 2. Buor-Khaya.

Position. Lena River delta, Kurungnah Island, Laptev Sea coast, northern Yakutia.

Age. Late Pleistocene.

Material. One p3, two dentary fragments, one postcranial bone.

Collector. T.V. Kuznetsova, 2000.

Locality 3. Bol'shoi Lyakhovskii Island.

Position. Laptev Sea, New Siberian Islands, northern Yakutia.

Age. Middle-Late Pleistocene.

Material. One skull fragment, 16 postcranial bones.

Collector. T.V. Kuznetsova, 1999.

Locality 4. Balyktakh.

Position. Kotel'nyi Island, Laptev Sea, northern Yakutia.

Age. Late Pleistocene.
Material. One P2, one skull fragment.

Collector. P.A. Nikol'skii.

Locality 5. Tordokh River.

Position. Novaya Sibir' Island, East Siberian Sea, northern Yakutia.

Age. Late Pleistocene.

Table 1. Anterior premolars measurements $(\mathrm{L}-$ length, W - width) in the Late Pleistocene Lepus tanaiticus from the East Siberian Arctic.

\begin{tabular}{|l|c|c|c|c|}
\hline Samples & LP2 & WP2 & Lp3 & Wp3 \\
\hline $\begin{array}{l}\text { Mamontovaya } \\
\text { Khayata }\end{array}$ & 2.6 & 2.2 & $\begin{array}{c}4.3-4.4 \\
.33 \pm 0.03 \\
\mathrm{n}=4\end{array}$ & $\begin{array}{c}3.3-4.3 \\
3.75 \pm 0.21 \\
\mathrm{n}=4\end{array}$ \\
\hline Buor-Khaya & & & 4.6 & 4.2 \\
\hline Balyktakh & 2.2 & 4.2 & & \\
\hline Svyatoi Nos & & & -4.3 & 3.7 \\
\hline Yana Paleolithic Site & & & 4.4 & 4.0 \\
\hline
\end{tabular}

Table 4. Scapula measurements in the Late Pleistocene Lepus tanaiticus from the East Siberian Arctic.

\begin{tabular}{|l|c|c|c|c|}
\hline Samples & S3 & S4 & S5 & S6 \\
\hline Mamontovaya Khayata & $8.3,8.4$ & 14.0 & 13.7 & $11.7,12.6$ \\
\hline Tordokh & & & 12.6 & 11.4 \\
\hline
\end{tabular}

Measurements: S3 - minimal length of scapular neck; S4 ventral end maximal length; S5 - glenoid anteroposterior diameter; S6 - glenoid width.

Table 2. Dentary measurements in the Late Pleistocene Lepus tanaiticus from the East Siberian Arctic.

\begin{tabular}{|l|c|c|c|c|c|c|c|}
\hline Samples & M1 & M2 & M3 & M4 & M5 & M6 & M7 \\
\hline $\begin{array}{l}\text { Mamontovaya } \\
\text { Khayata }\end{array}$ & 71.0 & 50.2 & $21.3,21.4$ & $\begin{array}{c}18.8-19.3 \\
19.05 \pm 0.25 \\
\mathrm{n}=4\end{array}$ & $\begin{array}{c}22.9-24.6 \\
23.58 \pm 0.38 \\
\mathrm{n}=4\end{array}$ & $\begin{array}{c}16.4-17.8 \\
17.10 \pm 0.40 \\
\mathrm{n}=3\end{array}$ & $\begin{array}{c}17.2-18.8 \\
\mathrm{n}=3\end{array}$ \\
\hline Buor-Khaya & & & 21.2 & 19.5 & 27.0 & 18.1 & 20.2 \\
\hline Svyatoi Nos* & 73.8 & 48.6 & $20.0,21.9$ & & $\begin{array}{c}21.2-25.1 \\
23.47 \pm 1.17 \\
\mathrm{n}=3\end{array}$ & $\begin{array}{c}15.4-17.6 \\
16.65 \pm 0.47 \\
\mathrm{n}=4\end{array}$ & $19.5,20.2$ \\
\hline $\begin{array}{l}\text { Yana } \\
\text { Paleolithic Site }\end{array}$ & & & 22.8 & & 25.7 & 17.5 & 19.2 \\
\hline
\end{tabular}

Measurements: M1 — dentary length; M2 - height of coronoid process; M3 - alveolar length of p3-m3; M4 — crown length of p3m3; M5 — diastema length; M6 - dentary depth at p3; M7 — dentary depth at m3.

* one specimen added from Averianov (1995).

Table 3. Skull measurements in the Late Pleistocene Lepus tanaiticus from the East Siberian Arctic.

\begin{tabular}{|l|c|c|c|c|c|c|c|c|c|c|c|c|c|}
\hline Samples & C4 & C5 & C6 & C7 & C8 & C10 & C11 & C12 & C13 & C14 & C16 & C18 & C19 \\
\hline Mamontovaya Khayata & 7.4 & 14.7 & 26.0 & 11.6 & 11.7 & 21.1 & 30.0 & 42.4 & 48.4 & 52.4 & 21.4 & 22.2 & 17.2 \\
\hline Bol'shoi Lyakhovskii Island & 8.0 & 14.8 & 24.4 & 11.2 & 10.3 & 19.6 & 29.2 & & & & & 24.0 & 18.2 \\
\hline Balyktakh & 9.1 & & & 9.1 & 11.4 & 21.1 & & & & & & & \\
\hline
\end{tabular}

Measurements: $\mathrm{C} 4$ - bony palate length; $\mathrm{C} 5$ - bony palate width between $\mathrm{P} 3$; $\mathrm{C} 6$ - incisive foramen length; $\mathrm{C} 7$ - incisive foramen width; C8 - choanae width; $\mathrm{C} 10$ - alveolar length of $\mathrm{P} 2-\mathrm{M} 3$; $\mathrm{C} 11$ - diastema length; $\mathrm{C} 12$ - zygomatic arch length; $\mathrm{C} 13$ - skull width at anterior zygomatic roots; $\mathrm{C} 14$ - skull width at posterior zygomatic roots; $\mathrm{C} 16$ - maximal nasal length along midline; $\mathrm{C} 18$ - frontals width anterior to supraorbital processes; $\mathrm{C} 19$ - frontals width posterior to supraorbital processes. 
Table 5. Humerus measurements in the Late Pleistocene Lepus tanaiticus from the East Siberian Arctic.

\begin{tabular}{|c|c|c|c|}
\hline Samples & \multicolumn{2}{|c|}{$\mathrm{H} 3$} & $\mathrm{H} 5$ \\
\hline Mamontovaya Khayata & & & $13.2,13.9$ \\
\hline $\begin{array}{l}\text { Bol'shoi Lyakhovskii } \\
\text { Island }\end{array}$ & & & $\begin{array}{c}13.0-14.5 \\
13.97 \pm 0.4 \\
8 n=3\end{array}$ \\
\hline Tordokh & \multicolumn{2}{|c|}{18.9} & \\
\hline Svyatoi Nos & & & $\begin{array}{c}12.8-13.9 \\
13.34 \pm 0.1 \\
8 \mathrm{n}=5\end{array}$ \\
\hline Yana Paleolithic Site & & & 12.3 \\
\hline \multicolumn{4}{|c|}{$\begin{array}{l}\text { Measurements: } \mathrm{H} 3 \text { - proximal end maximal width; } \mathrm{H} 5 \text { - dista } \\
\text { end width. }\end{array}$} \\
\hline \multicolumn{4}{|c|}{$\begin{array}{l}\text { Table 6. Radius measurements in the Late Pleistocene Lep- } \\
\text { us tanaiticus from the East Siberian Arctic. }\end{array}$} \\
\hline Samples & R1 & $\mathrm{R} 2$ & R3 \\
\hline Mamontovaya Khayata & 114.8 & $\begin{array}{c}8.7-10.3 \\
9.56 \pm 0.27 \\
n=5\end{array}$ & $10.3,10.7$ \\
\hline Bol'shoi Lyakhovskii Island & & & 10.6 \\
\hline Yana Paleolithic Site & & 9.5 & \\
\hline
\end{tabular}

Table 7. Ulna measurements in the Late Pleistocene Lepus tanaiticus from the East Siberian Arctic.

\begin{tabular}{|l|l|}
\hline Samples & U3 \\
\hline Mamontovaya Khayata & 9.8 \\
\hline Svyatoi Nos & 8.5 \\
\hline
\end{tabular}

Measurements: U3 - humerus articulation surface width.

Table 9. Pelvis measurements in the Late Pleistocene Lepus tanaiticus from the East Siberian Arctic.

\begin{tabular}{|l|c|c|c|c|}
\hline & P2 & P3 & P4 & P5 \\
\hline Mamontovaya & $15.2-17.6$ & & $18.7-21.7$ & $10.9-12.2$ \\
$16.48 \pm 0.50$ & & $\begin{array}{c}20.30 \pm 0.87 \\
\mathrm{n}=3\end{array}$ & $\begin{array}{c}11.55 \pm 0.35 \\
\mathrm{n}=3\end{array}$ \\
\hline Boyata & & $\mathrm{n}=4$ \\
Lyakhoi & $16.0-17.5$ & & $17.7-20.6$ & $11.0-12.7$ \\
Island & $16.94 \pm 0.21$ & 23.2 & $19.52 \pm 0.52$ & $11.91 \pm 0.20$ \\
$\mathrm{n}=8$ & & $\mathrm{n}=5$ & $\mathrm{n}=8$ \\
\hline & $16.0-16.8$ & & & $11.4-13.1$ \\
Svyatoi Nos & $16.33 \pm 0.24$ & & & $12.13 \pm 0.50$ \\
& $\mathrm{n}=3$ & & & $\mathrm{n}=3$ \\
\hline
\end{tabular}

Measurements: P2 - acetabulum length; P3 - symphysis length; P4 - obturator foramen length; P5 - minimal ilium height anterior to acetabulum.

Measurements: R1 - maximal length; R2 - proximal end width; R3 - distal end width.

Table 8. Metacarpals (II-IV) measurements in the Late Pleistocene Lepus tanaiticus from the East Siberian Arctic.

\begin{tabular}{|l|c|c|c|c|c|c|c|c|c|}
\hline Samples & LII & WpII & WdII & LIII & WpIII & WdIII & LIV & WpIV & WdIV \\
\hline Svyatoi Nos & $31.0,32.1$ & $4.4,4.5$ & $4.7,5.1$ & $29.6,29.9$ & $4.3,4.3$ & $4.6,4.8$ & 26.7 & 3.8 & 4.9 \\
\hline
\end{tabular}

Measurements: L — length; $\mathrm{Wp}$ - proximal end width; Wd — distal end width.

Table 10. Femur measurements in the Late Pleistocene Lepus tanaiticus from the East Siberian Arctic.

\begin{tabular}{|c|c|c|c|c|c|c|c|}
\hline Samples & $\mathrm{F} 1$ & $\mathrm{~F} 2$ & $\mathrm{~F} 3$ & $\mathrm{~F} 4$ & F5 & F6 & F7 \\
\hline $\begin{array}{l}\text { Mamontovaya } \\
\text { Khayata }\end{array}$ & 123.4 & 116.8 & $\begin{array}{c}27.1-28.6 \\
28.00 \pm 0.46 \\
n=3\end{array}$ & $\begin{array}{c}24.3-26.0 \\
25.25 \pm 0.36 \\
n=4\end{array}$ & $\begin{array}{c}10.3-11.1 \\
10.75 \pm 0.18 \\
n=4\end{array}$ & $\begin{array}{c}9.1-9.8 \\
9.35 \pm 0.16 \\
n=4\end{array}$ & $\begin{array}{c}19.6-21.0 \\
20.15 \pm 0.31 \\
n=4\end{array}$ \\
\hline Svyatoi Nos & & & & & & & 18.4 \\
\hline
\end{tabular}

Measurements: F1 — maximal length; F2 — distance from femoral head to distal end; F3 — proximal end width from femoral head to greater trochanter; F4 - proximal end width at level of lesser and third trochanters; F5 - femoral head anteroposterior diameter; F6 diaphysis minimal width; F7 - distal end width.

Table 11. Tibia measurements in the Late Pleistocene Lepus tanaiticus from the East Siberian Arctic.

\begin{tabular}{|l|c|c|c|c|c|}
\hline Samples & $\mathrm{T} 1$ & $\mathrm{~T} 2$ & $\mathrm{~T} 3$ & $\mathrm{~T} 4$ & $\mathrm{~T} 5$ \\
\hline Mamontovaya Khayata & 144.0 & 20.6 & $\begin{array}{c}7.18 \pm 0.23 \\
\mathrm{n}=4\end{array}$ & $\begin{array}{c}13.3-15.3 \\
14.54 \pm 0.28 \\
\mathrm{n}=8\end{array}$ & $\begin{array}{c}9.7-10.9 \\
10.30 \pm 0.13 \\
\mathrm{n}=8\end{array}$ \\
\hline Buor-Khaya & & & & 14.5 & 10.2 \\
\hline Bol'shoi Lyakhovskii Island & & & $8.2,8.5$ & $14.3,14.9$ & $9.8,9.9$ \\
\hline Tordokh & & 20.1 & & 15.3 & 10.8 \\
\hline Svyatoi Nos & & & & $14.4,15.3$ & $10.0,10.3$ \\
\hline Yana Paleolithic Site & & & & 14.1 & 10.1 \\
\hline
\end{tabular}

Measurements: T1 — maximal length; T2 - proximal end width; T3 — diaphysis minimal width; T4 — distal end width; T5 — distal end anteroposterior diameter. 
Material. Four postcranial bones.

Collector. P.A. Nikol'skii, 2002.

Locality 6. Cape Svyatoi Nos, Kondrat'eva River.

Position. Laptev Sea coast, northern Yakutia.

Age. Late Pleistocene.

Material. One p3, three dentary fragments, 31 postcranial bones.
Collector. P.A. Nikol'skii.

Locality 7. Yana River Paleolithic Site.

Position. Lower Yana River, northern Yakutia.

Age. Late Pleistocene.

Material. One p3, two dentary fragments, five postcranial bones.

Collector. P.A. Nikol'skii.

Table 12. Astragalus and calcaneus measurements in the Late Pleistocene Lepus tanaiticus from the East Siberian Arctic.

\begin{tabular}{|l|c|c|c|c|}
\hline Samples & $\mathrm{A} 1$ & $\mathrm{~A} 2$ & $\mathrm{C} 1$ & $\mathrm{C} 2$ \\
\hline Mamontovaya Khayata & & & 32.5 & 20.6 \\
\hline Bol'shoi Lyakhovskii Island & & & $31.6,33.0$ & $20.2,20.5$ \\
\hline \multirow{3}{*}{ Svyatoi Nos } & $17.2-18.0$ & $7.2-9.0$ & $33.2-37.2$ & $20.7-23.3$ \\
& $17.70 \pm 0.19$ & $8.01 \pm 0.25$ & $34.38 \pm 0.95$ & $21.55 \pm 0.60$ \\
$\mathrm{n}=4$ & $\mathrm{n}=6$ & $\mathrm{n}=4$ & $\mathrm{n}=4$ \\
\hline
\end{tabular}

Measurements: A1 — astragalus maximal length; A2 — astragalus trochlea width; $\mathrm{C} 1$ — calcaneum maximal length; $\mathrm{C} 2$ — distance from tuber calcanei proximal end to fibular facet distal end.

Table 13. Metatarsals (II-V) measurements in the Late Pleistocene Lepus tanaiticus from the East Siberian Arctic.

\begin{tabular}{|l|c|c|c|c|c|c|c|c|c|c|c|c|}
\hline Samples & LII & WpII & WdII & LIII & WpIII & WdIII & LIV & WpIV & WdIV & LV & WpV & WdV \\
\hline Mamontovaya Khayata & & 4.7 & & & & & & & & & & \\
\hline Bol'shoi Lyakhovskii Island & 49.9 & 4.9 & 6.4 & 53.0 & 5.4 & 6.4 & & & & & & \\
\hline Tordokh & & & & 52.3 & 5.5 & 6.4 & & & & & & \\
\hline Svyatoi Nos & & & & 53.5 & 5.7 & 6.7 & $52.2,52.7$ & $6.0,6.2$ & $5.9,6.4$ & 48.6 & 8.5 & 5.6 \\
\hline Yana & & & & 53.0 & 5.2 & 6.3 & & & & 43.2 & 8.5 & 5.5 \\
\hline
\end{tabular}

Measurements: L — length; Wp — proximal end width; Wd — distal end width.

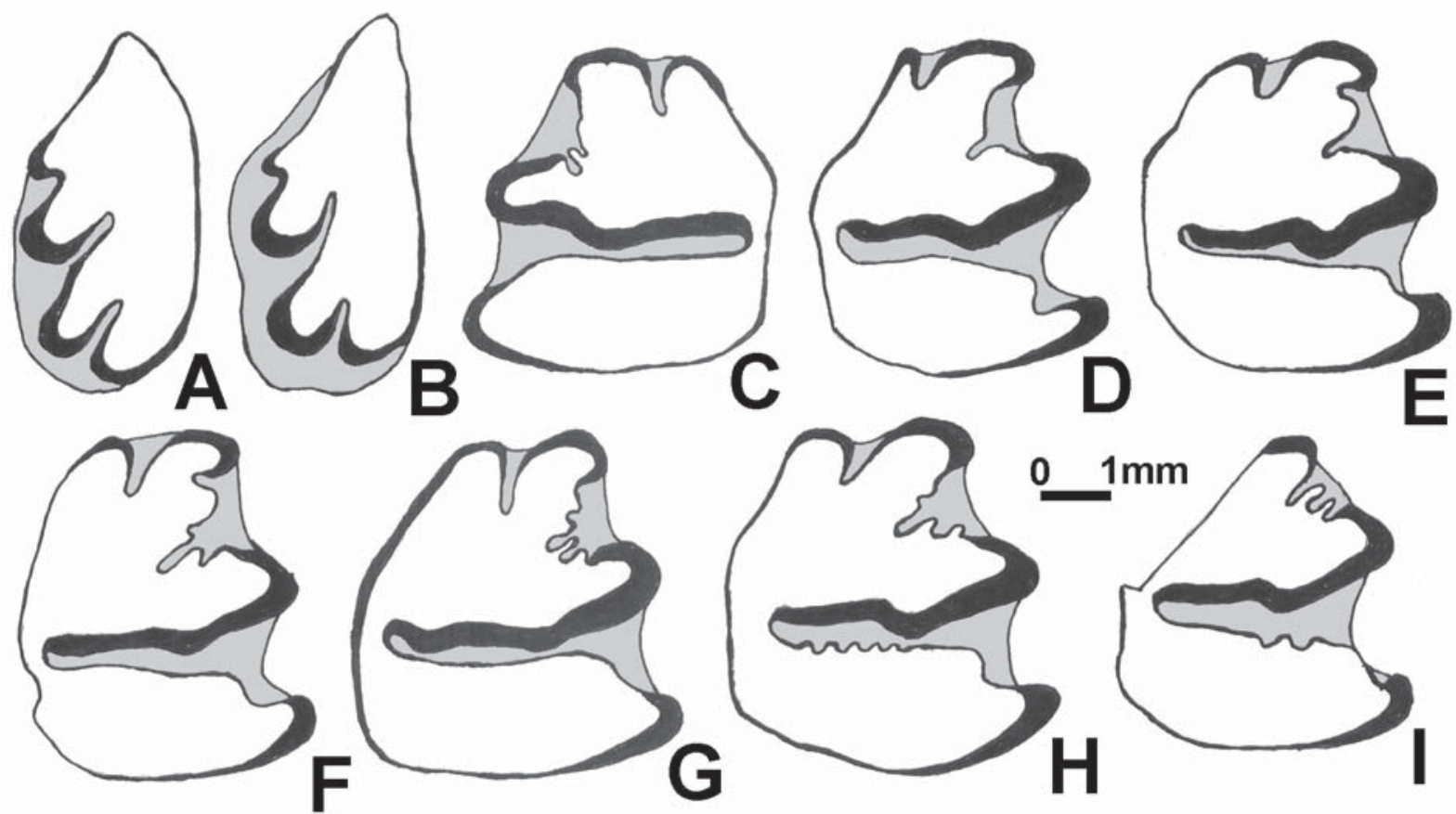

Figure 2. P2 (A, B) and p3 (C-I) of Lepus tanaiticus from the East Siberian Arctic in occlusal view. Dentine is white, enamel is black, and cement is gray. Scale bar is $1 \mathrm{~mm}$.

A, C-F - Mamontovaya Khayata, PIN MKh-O276 (left), MKh00-O115 (left), MKh-O83 (right), MKh-O164 (right), and MKh-O82 (right); B - Balyktakh, PIN without number (left); G — Buor-Khaya, PIN BKh-O93 (right); H — Yana River Paleolithic Site, PIN without number (right); I - Svyatoi Nos, PIN without number (right incomplete). 


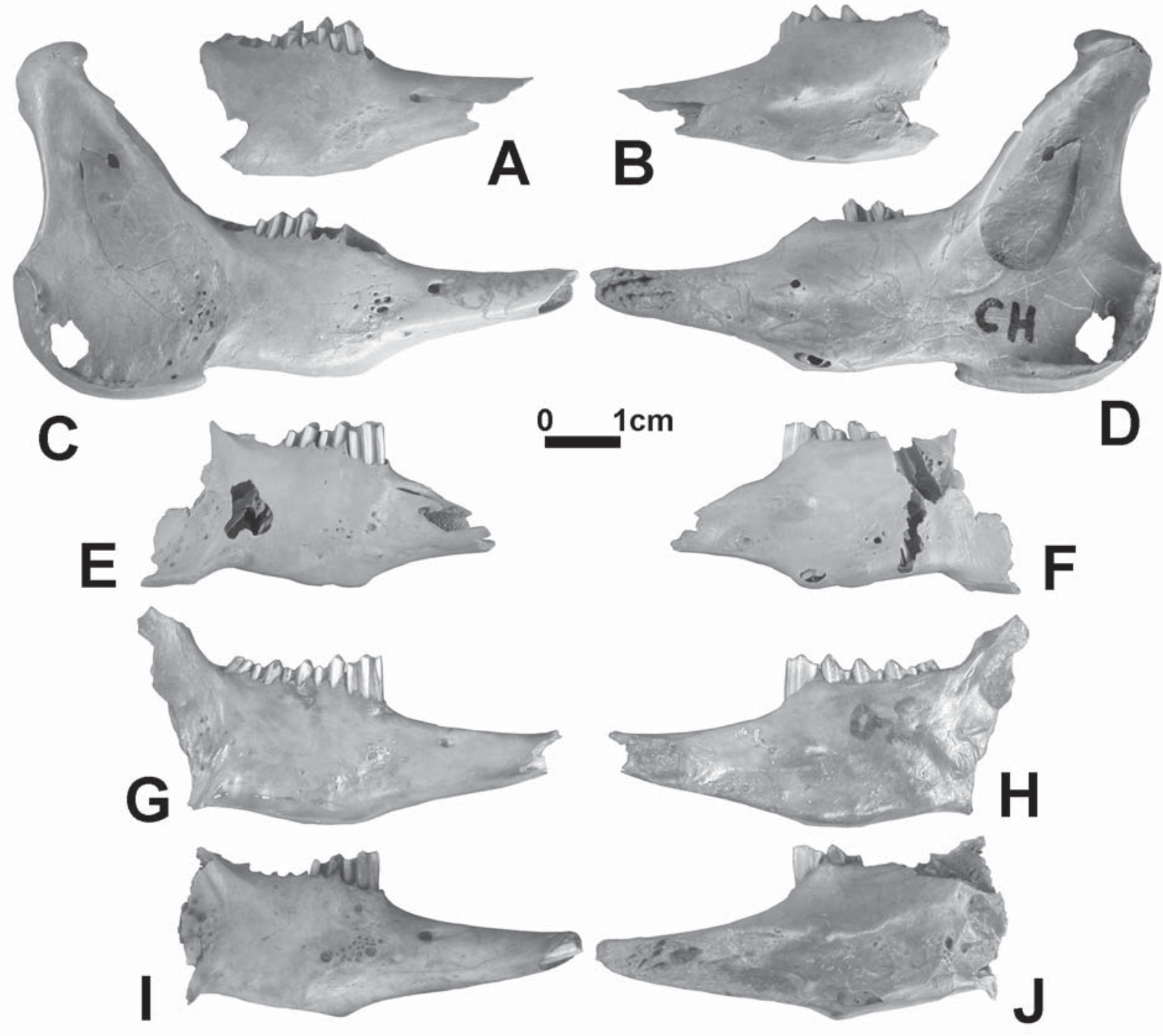

Figure 3. Right dentaries of Lepus tanaiticus from the East Siberian Arctic in labial (A, C, E, G, I) and lingual (B, D, F, H, J) views. Scale bar is $1 \mathrm{~cm}$.

A, B - Svyatoi Nos, PIN Sv-Nos 1; C, D - Svyatoi Nos, PIN Sv-Nos 2; E, F — Mamontovaya Khayata, PIN MKh-O164; G, H Mamontovaya Khayata, PIN MKh-O83; I, J — Yana River Paleolithic Site, PIN without number.

\section{Description and discussion}

New Pleistocene hare materials from the East Siberian Arctic agrees well with the description of L. tanaiticus (Gureev, 1964; Averianov \& Kuz'mina, 1993; Averianov, 1999): they similarly large in size (Tabs. 1-13), have complicated $\mathrm{p} 3$ morphology with larger number of minute folds in the anteroexternal reentrant (Fig. 2; 1-5 folds, $\mathrm{M}=3.0 \pm 0.53, \mathrm{n}=7)$; in L. tanaiticus this mean number exceeds 2.0 in contrast with the Recent and Pleistocene L. timidus populations, where it varies from 0.50 to 1.79 , see Averianov, 1999: tab.7), and have very deep horizontal ramus of the dentary (Fig. 3). Two p3 specimens (Fig. 2H, I) have folded anterior talonid margin, which is usually not characteristic for the white hares lineage.
In size the Pleistocene L. tanaiticus exceeds most of the modern white hares populations, except the largest Arctic subspecies L. timidus tschuktschorum Nordquist, 1883, which approximates the smallest samples of $L$. tanaiticus (Fig. 4). Hares from Mamontovaya Khayata are almost identical in p3 and dentary measurements with the sample from Berelekh, and could be confidently attributed to L. tanaiticus vereschagini described from that site (Averianov, 1995). Materials from other East Siberian Arctic localities are still insufficient for the subspecies determination. Larger specimens from BuorKhaya and Yana Paleolithic Site may indicate existence of a distinct subspecies.

ACKNOWLEDGEMENTS. We are grateful to Dr. Alexei S. Tesakov for comments on the manuscript. This research was supported by the Russian Science Support Foundation. 


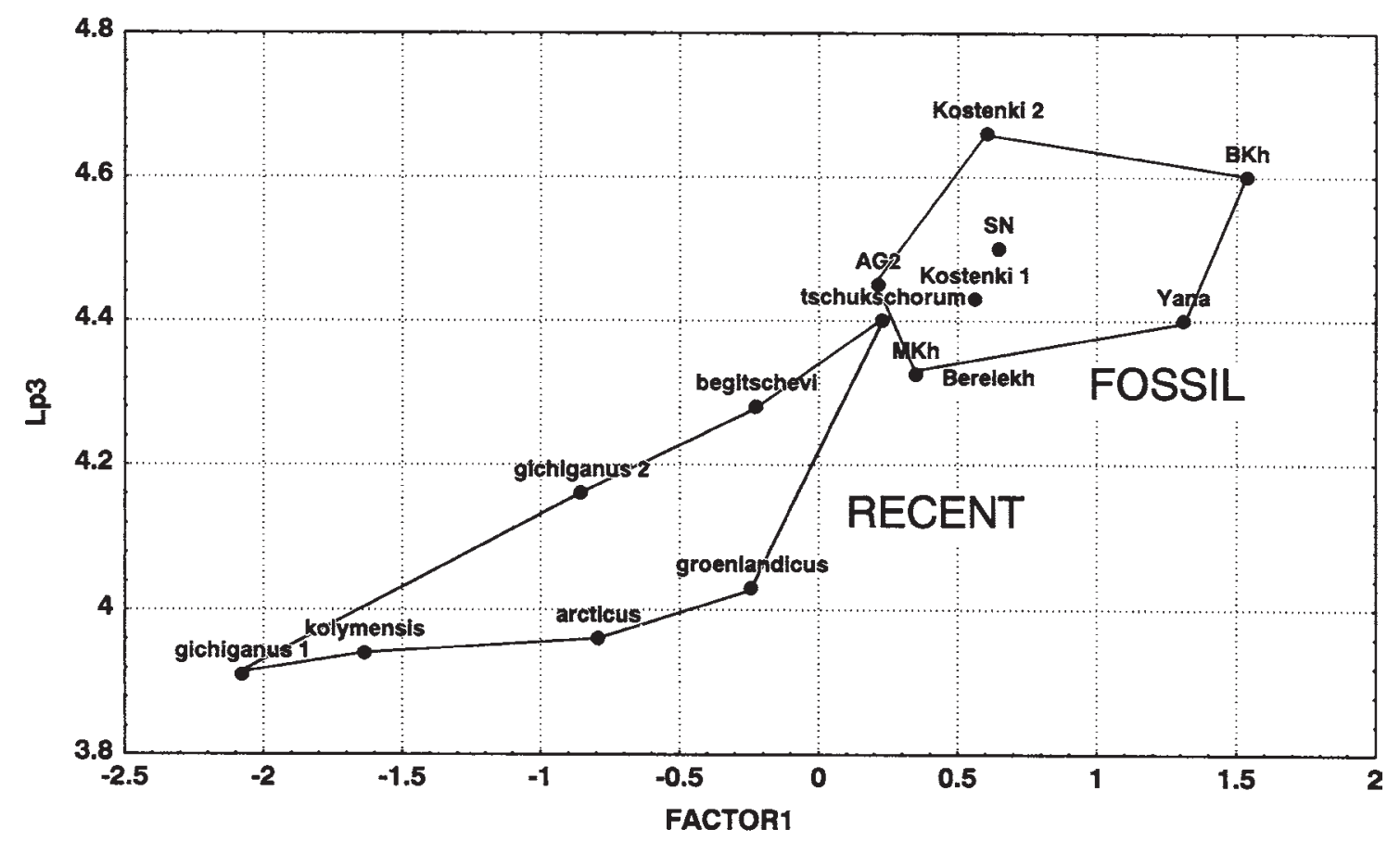

Figure 4. Scatterplot diagram of the $\mathrm{p} 3$ length (Lp3) relation to the dentary measurements exemplified by FACTOR 1 from the principal component analysis in the recent and Pleistocene Lepus samples (delineated separately). Measurements are from Averianov (1995) and this paper.

Recent samples: L. timidus gichiganus J.A.Allen, 1903 (1 - Yakutia, 2 - Chukotka); L. timidus kolymensis Ognev, 1922 (eastern Yakutia); L. timidus begitschevi Koljuschev, 1936 (Taimyr); L. timidus tschuktschorum Nordquist, 1883 (eastern Chukotka); L. arcticus Ross, 1819 (Alaska and Canada).

Fossil samples: Mamontovaya Khayata (MKh, Yakutia); Berelekh (Yakutia, type locality for L. tanaiticus vereschagini Averianov, 1995); Afontova Gora 2 (AG2, Krasnoyarsk Territory); Kostenki (Voronezh Province, 1 - humus deposits, type locality for L. tanaiticus gmelini Averianov \& Kuz'mina, 1993; 2 - loess deposits, type locality for L. tanaiticus tanaiticus Gureev, 1964); Svyatoi Nos (SN, Yakutia); Yana River Paleolithic Site (Yakutia); Buor-Khaya (BKh).

\section{References}

Averianov A.O. 1995. [Late Pleistocene hare, Lepus tanaiticus (Lagomorpha, Leporidae) of Siberia] // Trudy Zoologicheskogo Instituta AN SSSR. T.263. P.121-162 [in Russian].

Averianov A.O. 1999. Late Pleistocene hares (Lepus) of the Russian Plain // Saunders J.J., Styles B.W. \& Baryshnikov G.F. (eds.). Quaternary Paleozoology in the Northern Hemisphere. Illinois State Museum Scientific Papers. Vol.27. P.40-68.

Averianov A.O. \& Kuz'mina I.E. 1993. [Don hare, Lepus tanaiticus Gureev, 1964 from the Paleolithic sites Kostenki] // Trudy Zoologicheskogo Instituta AN SSSR. T.249. P.66-92 [in Russian].
Gureev A.A. 1964. [Lagomorphs (Lagomorpha). Fauna of the USSR. Mammals]. T.3. Vyp.10. Moskva-Leningrad: Nauka. 276 p. [in Russian].

Kuz'mina S., Kuznetsova T. \& Sher A. 1999. Paleontological research on the Bykovsky Peninsula // Berichte zur Polarforschung. No.315. P.179-187, 227-259.

Kuznetsova T. \& Kuz'mina S. 2000. Paleontological research at the southern coast of Bol'shoy Lyakhovsky Island // Berichte zur Polarforschung. No.354. P.151-161, 223258.

Kuznetsova T. \& Kuz'mina S. 2001. Paleontological research of the western Olenyok Channel - Nagym, Kurungnakh Island (Buor Khaya) // Berichte zur Polarforschung. No.388. P.92-93, 96-97, 113, 123-127, 131. 